Research Article

\title{
Prediction Method of Cavitation Jet Wave Attenuation Based on Five-Equation Two-Fluid Model
}

\author{
You Fu $(\mathbb{D}$, Zhaopeng Xie, and Weiguo Zhao \\ School of Energy and Power Engineering, Lanzhou University of Technology, Lanzhou 730050, China \\ Correspondence should be addressed to You Fu; fuyou_894513@hotmail.com
}

Received 8 November 2019; Accepted 16 January 2020; Published 11 February 2020

Academic Editor: Zhengbiao Peng

Copyright $\odot 2020$ You Fu et al. This is an open access article distributed under the Creative Commons Attribution License, which permits unrestricted use, distribution, and reproduction in any medium, provided the original work is properly cited.

\begin{abstract}
In this paper, the seven-equation two-fluid flow model is streamlined to a five-equation numerical calculation method. This method is applied to predict the wave attenuation of the cavitation jet. Compared with 9 different experimental schemes in two separate laboratories, it is found that the velocity flow characteristics are different from normal transient flow when the cavitation jet is formed in the pipeline. Such a difference in velocity flow characteristics will cause changes in the prediction deviation of pressure response time and amplitude. A numerical method for predicting the attenuation of pressure fluctuation with the cavitation jet is presented taken into account these flow characteristics. In this method, the unsteady friction model is opened after the jet wave transmits 3-5 cycles, and the cavitation jet wave attenuation is well simulated. This calculation method could provide a research basis for pipeline leakage, vibration, noise, and other fields that need accurate pressure signals.
\end{abstract}

\section{Introduction}

Water hammer with column separation in a pipeline system can cause cavitation jet [1-3]. The formation of the cavitation jet will cause a sharp rise in pressure, which has a serious impact on the safety of the pipeline system [4-8]. In recent years, scholars have begun to use the two-fluid model to study the cavitation jet phenomenon both in time-effectiveness and calculation accuracy in the pipeline system [9-14]. A typical two-fluid two-phase flow model under one-dimensional direction requires the solution of 7-9 equations [15-23]. At the same time, the cavitation jet which is a typical transient flow phenomenon usually calculated in an explicit format. The complexity of the calculation model and solution format takes more computation time. Therefore, this method sometimes is not appropriate for engineering applications.

There are two main factors influencing the calculation accuracy of water hammer cavitation jet in the pipeline system: (1) residual void of gas and (2) wave attenuation. Residual void of gas means that the volume of initial gas increases with the progress of time iteration. The principle of this phenomenon is that the initial calculation needs a certain amount of insoluble gas to satisfy the appeared second phase (water vapor) in the two-fluid model. The energy loss is not considered in the interaction between the two phases by the two-fluid model in theory. The expansion value of bubbles is often larger than the compression, which is expected to result in the void of gas gradually increasing with the advance of time. So the expansion and compression stages of the bubbles are unbalanced under the condition of constant relaxation coefficient in real calculation [19-21, 23], resulting in the residual void of gas. The residual void of gas will cause an obvious influence on the velocity of wave transmission. It will continuously affect the wave, which will lead to obvious errors in the prediction of water hammer wave.

Wave attenuation refers to the attenuation of the pressure wave and the degree of energy dissipation with the advance of time, which is also an important part of transient research [24-32]. Accurate prediction of water hammer cavitation jet in a pipeline system is the basis of prediction of pipeline obstruction and leakage. The main problem of wave attenuation lies in the combination of unsteady friction model and cavitation jet calculation method. The two decay coefficient models added into the two-fluid model will take such solution process more complicated. When the large intensity of the cavitation jet is generated, the applicability of the unsteady friction model with the two-fluid model has been rarely reported in the literature. Therefore, it is an 
urgent problem to predict the wave attenuation of the cavitation jet.

Another factor is the timeliness of the calculations. The calculation of the cavitation jet in the pipeline system is generally isentropic flow. The energy equation in this calculation model is used to represent the energy change between phases when the relaxation phenomenon happened. However, the change of energy between phases is mainly reflected in the correction of two-phase pressure, which results in the waste of computing resources.

This paper simplified the two-phase flow model with seven equations to a numerical calculation method with five equations. A numerical method for predicting the attenuation of pressure fluctuation with the cavitation jet is presented. This method is applied to predict the wave attenuation of the cavitation jet. The main contents are as follows:

(1) The calculation method of velocity and pressure relaxation coefficient is rederived strictly through the interphase action mechanism of the seven-equation two-fluid model.

(2) A five-equation calculation method is constructed.

(3) The internal flow parameters were analyzed to reveal the internal flow trend of the cavitation jet.

(4) By setting the internal switch, the two-coefficient decay model was added into the five-equation calculation method. The calculation method of wave attenuation suitable for water jet cavitation was constructed.

\section{Two-Phase Flow Model and Properties}

2.1. Two-Fluid Model. The two-fluid model usually consists of 7-8 equations. Each phase has its density, velocity, and pressure. The two-phase equations are coupled by the interphase forces and relaxation coefficients. In the macroscopic Euler-Euler model, the microscopic parameters in the macroscopic model can be described by adding the void transport equation and the number of bubble concentration equation. The numerical calculation model selected in this paper is shown in the following equations:

$$
\begin{gathered}
\frac{\partial \alpha_{1}}{\partial t}+u_{I} \nabla \alpha_{1}=\mu\left(P_{1}-P_{2}\right) \\
\frac{\partial \alpha_{k} \rho_{k}}{\partial t}+\nabla \cdot\left(\alpha_{k} \rho_{k} u_{k}\right)=0 \\
\frac{\partial \alpha_{k} \rho_{k} u_{k}}{\partial t}+\nabla \cdot\left(\alpha_{k} \rho_{k} u_{k}^{2}+\alpha_{k} P_{k}\right)=P_{I} \nabla \alpha_{k} \pm \lambda\left(u_{2}-u_{1}\right) \\
\frac{\partial \alpha_{k} \rho_{k} E_{k}}{\partial t}+\nabla \cdot\left(\alpha_{k} \rho_{k} u_{k} E_{k}+\alpha_{k} P_{k} u_{k}\right)=P_{I} u_{I} \nabla \alpha_{k} \\
\pm \mu \overline{P_{I}}\left(P_{2}-P_{1}\right) \pm \lambda \overline{u_{I}}\left(u_{2}-u_{1}\right)-K_{k g f} u_{k}
\end{gathered}
$$

where the subscript $k$ is 1 and 2, in which 1 is the gas phase and 2 is the liquid phase; $I$ is the interphase; $\alpha$ is the void fraction; $u$ is the velocity, $\mathrm{m} / \mathrm{s} ; P$ is the pressure, $\mathrm{Pa} ; \rho$ is the density, $\mathrm{kg} / \mathrm{m}^{3} ; E$ is the total energy $E=e+1 / 2 u^{2}, \mathrm{~J}$; $\mu$ is the pressure relaxation coefficient; $\lambda$ is the velocity relaxation coefficient; and $K_{\mathrm{kgf}}$ is the gravity and resistance term.

The calculation method of the interphase boundary was referred to the literature $[15,16]$. The expression equation is as follows:

$$
\begin{aligned}
& P_{I}=\overline{P_{I}}=\alpha_{1} P_{1}+\alpha_{2} P_{2}, \\
& u_{I}=\overline{u_{I}}=\frac{\alpha_{1} \rho_{1} u_{1}+\alpha_{2} \rho_{2} u_{2}}{\alpha_{1} \rho_{1}+\alpha_{2} \rho_{2}} .
\end{aligned}
$$

2.2. Conventional Closure Relation. The equation of state (EOS) was introduced for the closure of the equation in this model. The function of the EOS is to create a relationship between internal energy, pressure, and density to transform the model.

This paper cited a modified form of the stiffened gas in Suarel [18] and Crouzet [12]. The expression for this equation of state is as follows:

$$
e_{k}\left(p_{k}, \rho_{k}\right)=\frac{p_{k}+\gamma_{k} \pi_{k}}{\rho_{k}\left(\gamma_{k}-1\right)}+q_{k}, \quad k=1,2,
$$

where $\gamma_{k}$ is the specific heat ratio, which is $\gamma_{k}=C_{p, k} / C_{v, k} \cdot \pi_{1}$ usually takes the value of 0 . The expression for $\pi_{2}$ is as follows:

$$
\pi_{2}=\rho_{2}\left(T_{\text {ref }}\right)\left(\gamma_{2}-1\right) C_{v, 2} T_{\text {ref }}-p_{\text {sat }}\left(T_{\text {ref }}\right),
$$

where $T_{\text {ref }}$ is the experimental ambient temperature. $p_{\text {sat }}$ is the saturated vapor pressure.

2.3. Improved Relaxation Calculation Method. Relaxation refers to a process in the transient flow where two phases tend to balance between each other [19-21, 23]. In transient flow, as the parameters of two-phase are usually different, the velocity of the wave will be greatly deviated. After each time of transients, the two phases need an equilibrium process to end the difference caused by the transient flow. The relaxation coefficient is an empirical coefficient used to calculate the relaxation process.

The construction methods of relaxation coefficient are divided into finite and infinite relaxation coefficient construction methods. In this paper, a new construction method of relaxation coefficients is established by combining the two kinds of relaxation coefficients.

During the relaxation process, it is considered that the development of this process is very short, and some literatures indicate that the development process is nearly $1 \mathrm{~ms}$. In the process of interphase relaxation, the influence of spatial variables on the relaxation process can be ignored, and only the change of the partial derivative of the time term is reckoned. Therefore, equations (1)-(4) were sorted out, ignoring the space term and the source term independent of the relaxation process. The equations are modified into the following form: 


$$
\begin{aligned}
& \frac{\partial \alpha_{1}}{\partial t}=\mu\left(P_{1}-P_{2}\right), \\
& \frac{\partial P_{1}}{\partial t}=\frac{\mu\left(P_{2}-P_{1}\right)\left(P_{I}-\rho_{1}^{2} \partial e_{1} / \partial \rho_{1}\right)}{\alpha_{1} \rho_{1} \partial e_{1} / \partial P_{1}}, \\
& \frac{\partial P_{2}}{\partial t}=\frac{\mu\left(P_{1}-P_{2}\right)\left(P_{I}-\rho_{2}^{2} \partial e_{2} / \partial \rho_{2}\right)}{\alpha_{2} \rho_{2} \partial e_{2} / \partial P_{2}} .
\end{aligned}
$$

Equations (9)-(11) could be converted to (26)-(28):

$$
\begin{aligned}
& \alpha_{1}^{*}-\alpha_{1}^{0}=\mu\left(P_{1}-P_{2}\right) \Delta t, \\
& P_{1}^{*}-P_{1}^{0}=\frac{\mu\left(P_{2}-P_{1}\right)\left(P_{I}-\rho_{1}^{2} \partial e_{1} / \partial \rho_{1}\right)}{\alpha_{1} \rho_{1} \partial e_{1} / \partial P_{1}} \Delta t,
\end{aligned}
$$

$$
P_{2}^{*}-P_{2}^{0}=\frac{\mu\left(P_{1}-P_{2}\right)\left(P_{I}-\rho_{2}^{2} \partial e_{2} / \partial \rho_{2}\right)}{\alpha_{2} \rho_{2} \partial e_{2} / \partial P_{2}} \Delta t,
$$

where the variables marked with * indicate the relaxed ones and the variables marked with 0 are the calculated ones. Relaxation process is an interphase equilibrium process. Thus at the end of the relaxation process, there should be $P_{1}^{*}-P_{2}^{*}=-2 S / R$ or be a tendency to $-2 S / R$. By subtracting equation (13) from equation (14) and substituting $P_{1}^{*}-P_{2}^{*}=$ $-2 S / R$ into the original system of equations, the following expression can be obtained:

$$
\begin{aligned}
& P_{1}^{*}-P_{2}^{*}=\left(P_{1}^{0}-P_{2}^{0}\right)\left\{1+\Delta t \mu\left[\frac{\left(P_{I}-\rho_{2}^{2} \partial e_{2} / \partial \rho_{2}\right)}{\alpha_{2} \rho_{2} \partial e_{2} / \partial P_{2}}-\frac{\left(P_{I}-\rho_{1}^{2} \partial e_{1} / \partial \rho_{1}\right)}{\alpha_{1} \rho_{1} \partial e_{1} / \partial P_{1}}\right]\right\}, \\
& P_{1}^{*}-P_{2}^{*}=\frac{-2 S}{R}, \\
& \left(P_{1}^{0}-P_{2}^{0}\right)\left\{1+\Delta t \mu\left[\frac{\left(P_{I}-\rho_{2}^{2} \partial e_{2} / \partial \rho_{2}\right)}{\alpha_{2} \rho_{2} \partial e_{2} / \partial P_{2}}-\frac{\left(P_{I}-\rho_{1}^{2} \partial e_{1} / \partial \rho_{1}\right)}{\alpha_{1} \rho_{1} \partial e_{1} / \partial P_{1}}\right]\right\}=-\frac{2 S}{R}
\end{aligned}
$$

The relaxation process of pressure is caused by $\left(P_{2}^{0}-P_{1}^{0}\right) \neq 0$. So the following formulas are required to achieve the entire relaxation process:

$$
\begin{aligned}
1+\Delta t \mu\left[\frac{\left(P_{I}-\rho_{2}^{2} \partial e_{2} / \partial \rho_{2}\right)}{\alpha_{2} \rho_{2} \partial e_{2} / \partial P_{2}}-\frac{\left(P_{I}-\rho_{1}^{2} \partial e_{1} / \partial \rho_{1}\right)}{\alpha_{1} \rho_{1} \partial e_{1} / \partial P_{1}}\right]=-\frac{2 S}{R\left(P_{1}^{0}-P_{2}^{0}\right)}, \\
\mu\left[\frac{\left(P_{I}-\rho_{2}^{2} \partial e_{2} / \partial \rho_{2}\right)}{\alpha_{2} \rho_{2} \partial e_{2} / \partial P_{2}}-\frac{\left(P_{I}-\rho_{1}^{2} \partial e_{1} / \partial \rho_{1}\right)}{\alpha_{1} \rho_{1} \partial e_{1} / \partial P_{1}}\right]=\left[-\frac{2 S}{R\left(P_{1}^{0}-P_{2}^{0}\right)}-1\right] \frac{1}{\Delta t} .
\end{aligned}
$$

Further finishing could be obtained as follows:

$$
\begin{aligned}
\mu & =-\frac{\left(2 S / R\left(P_{1}^{0}-P_{2}^{0}\right)\right)+1}{\left[\left(\left(P_{I}-\rho_{2}^{2} \partial e_{2} / \partial \rho_{2}\right) /\left(\alpha_{2} \rho_{2} \partial e_{2} / \partial P_{2}\right)\right)-\left(P_{I}-\rho_{1}^{2} \partial e_{1} / \partial \rho_{1}\right) /\left(\alpha_{1} \rho_{1} \partial e_{1} / \partial P_{1}\right)\right] \Delta t}, \\
P_{I} & =\alpha_{1} P_{1}+\alpha_{2} P_{2}, \\
\frac{2 S}{R} & \approx 1 \sim 5 \times 10^{4}, \quad \Delta t \approx 1 \times 10^{-5} .
\end{aligned}
$$

For the velocity relaxation method, as the relaxation phenomenon is independent of the spatial variables and is only a function in the time direction, the original equations were transformed into the following equations:

$$
\begin{gathered}
\frac{\partial \alpha_{1} \rho_{1} u_{1}}{\partial t}=\lambda\left(u_{2}-u_{1}\right), \\
\frac{\partial \alpha_{2} \rho_{2} u_{2}}{\partial t}=-\lambda\left(u_{2}-u_{1}\right),
\end{gathered}
$$

$$
\begin{aligned}
& \frac{\partial u_{1}}{\partial t}=\frac{\lambda\left(u_{2}-u_{1}\right)}{\alpha_{1} \rho_{1}}, \\
& \frac{\partial u_{2}}{\partial t}=\frac{-\lambda\left(u_{2}-u_{1}\right)}{\alpha_{2} \rho_{2}} .
\end{aligned}
$$

The equation could be transformed as follows: 


$$
\begin{aligned}
& u_{1}^{*}-u_{1}^{0}=\frac{\Delta t \lambda\left(u_{2}-u_{1}\right)}{\alpha_{1} \rho_{1}}, \\
& u_{2}^{*}-u_{2}^{0}=\frac{-\Delta t \lambda\left(u_{2}-u_{1}\right)}{\alpha_{2} \rho_{2}} .
\end{aligned}
$$

Further finishing could be obtained as follows:

$$
\begin{aligned}
& u_{1}^{*}=\frac{\Delta t \lambda\left(u_{2}-u_{1}\right)}{\alpha_{1} \rho_{1}}+u_{1}^{0}, \\
& u_{2}^{*}=\frac{-\Delta t \lambda\left(u_{2}-u_{1}\right)}{\alpha_{2} \rho_{2}}+u_{2}^{0} .
\end{aligned}
$$

It is desirable to the relaxation state $u_{1}^{*}-u_{2}^{*}=0$ or tends to 0 during the transient process. Subtracting the above two equations gives the following expressions:

$$
\begin{aligned}
& u_{1}^{*}-u_{2}^{*}=\frac{\Delta t \lambda\left(u_{2}-u_{1}\right)}{\alpha_{1} \rho_{1}}+\frac{\Delta t \lambda\left(u_{2}-u_{1}\right)}{\alpha_{2} \rho_{2}}+u_{1}^{0}-u_{2}^{0}, \\
& \frac{\Delta t \lambda\left(u_{2}-u_{1}\right)}{\alpha_{1} \rho_{1}}+\frac{\Delta t \lambda\left(u_{2}-u_{1}\right)}{\alpha_{2} \rho_{2}}+u_{1}^{0}-u_{2}^{0}=0, \\
& \lambda=\left[\Delta t\left(\frac{1}{\alpha_{1} \rho_{1}}+\frac{1}{\alpha_{2} \rho_{2}}\right)\right]^{-1} .
\end{aligned}
$$

Therefore, equation (33) is an expression for the improved calculation of the velocity relaxation method.

\section{Simplified Calculation Model}

When the model (1)-(4) is used for calculation, it is usually settled as isentropic flow. The equation of energy is primarily used to correct the imbalance of pressure between two phases. When the expression of pressure relaxation coefficient is obtained, the energy equation for the gas and liquid phases can be omitted. The model can be solved only by the void transport equation, continuity equations, and momentum equations. Some literature has pointed out that the velocity of two phases in the two-fluid model can be considered as negligible when the void of the fraction of the gas phase is not large [33]. So the equation can be further simplified as follows:

$$
\begin{aligned}
\frac{\partial \alpha_{1}}{\partial t}+u \nabla \alpha_{1} & =\mu\left(P_{1}-P_{2}\right), \\
\frac{\partial \alpha_{k} \rho_{k}}{\partial t}+\nabla \cdot\left(\alpha_{k} \rho_{k} u\right) & =0 \\
\frac{\partial \alpha_{k} \rho_{k} u}{\partial t}+\nabla \cdot\left(\alpha_{k} \rho_{k} u^{2}+\alpha_{k} P_{k}\right) & =P_{I} \nabla \alpha_{k} .
\end{aligned}
$$

The density term in the equation can be transformed to the pressure through the relation between density and pressure (37) and (38):

$$
\begin{aligned}
\frac{\mathrm{d} \rho_{k}}{\mathrm{~d} t} & =\frac{r_{k}}{c_{k}^{2}} \frac{\mathrm{d} P_{k}}{\mathrm{~d} t}, \\
\rho_{k}^{*} & =\rho_{k}^{0}+\frac{r_{k}}{c_{k}^{2}}\left(P_{k}^{*}-P_{k}^{0}\right) .
\end{aligned}
$$

The equations could be simplified into the following onedimensional form to solve the cavitation jet in the pipeline:

$$
\begin{gathered}
\frac{\partial \alpha_{1}}{\partial t}+u \frac{\partial \alpha_{1}}{\partial x}=\mu\left(P_{1}-P_{2}\right), \\
\frac{\partial P_{1}}{\partial t}+u \frac{\partial P}{\partial t}+\frac{\rho_{1} c_{1}^{2}}{r_{1}} \frac{\partial u}{\partial x}=\frac{c_{1}^{2} \rho_{1} \mu\left(P_{2}-P_{1}\right)}{r_{1} \alpha_{1}} \\
\frac{\partial P_{2}}{\partial t}+u \frac{\partial P_{2}}{\partial t}+\rho_{2} c_{2}^{2} \frac{\partial u}{\partial x}=\frac{c_{2}^{2} \rho_{2} \mu\left(P_{1}-P_{2}\right)}{r_{2} \alpha_{2}}, \\
\frac{\partial u}{\partial t}+u \frac{\partial u}{\partial t}+\frac{1}{\rho_{1}} \frac{\partial P_{1}}{\partial x}+\frac{P_{1}-P_{I}}{\alpha_{1} \rho_{1}} \frac{\partial \alpha_{1}}{\partial x}=-\frac{K_{1 \mathrm{gf}}}{\alpha_{1} \rho_{1}} \\
\frac{\partial u}{\partial t}+u \frac{\partial u}{\partial t}+\frac{1}{\rho_{2}} \frac{\partial P_{2}}{\partial x}+\frac{P_{I}-P_{2}}{\alpha_{2} \rho_{2}} \frac{\partial \alpha_{1}}{\partial x}=-\frac{K_{2 \mathrm{gf}}}{\alpha_{2} \rho_{2}}
\end{gathered}
$$

The system of equations has five distinct eigenvalues, and the equation is unconditional hyperbolic:

$$
\lambda_{1}=u \lambda_{2}=u+\sqrt{\frac{c_{2}^{2}}{r_{2}}} \lambda_{3}=u-\sqrt{\frac{c_{2}^{2}}{r_{2}}} \lambda_{4}=u+\sqrt{\frac{c_{1}^{2}}{r_{1}}} \lambda_{5}=u-\sqrt{\frac{c_{1}^{2}}{r_{1}}},
$$

where $c_{k}$ represents the wave velocity, and the expression is shown as follows:

$$
c_{k}^{2}=\frac{P_{k} / \rho_{k}^{2}-\partial e_{k} / \partial \rho_{k}}{\partial e_{k} / \partial P_{k}} .
$$

\section{Numerical Resolution}

4.1. Hyperbolic Operator. The equations can be written as follows:

$$
\frac{\partial \phi}{\partial t}+\frac{\partial f(\phi)}{\partial x}=S
$$

The parameter of (46) could be expressed as follows:

$$
\frac{\partial f}{\partial \phi}=a, \quad a=a^{+}+a^{-} a^{+}=\frac{1}{2}(a+|a|) a^{-}=\frac{1}{2}(a-|a|),
$$

$$
f=a^{+} \phi+a^{-} \phi=f^{+}+f^{-} \quad \frac{\partial \phi}{\partial t}+\frac{\partial f^{+}}{\partial x}+\frac{\partial f^{-}}{\partial x}=S .
$$

The numerical calculation method in NND format (49) can be obtained by further sorting out these equations.

The NND format is a nonoscillatory, nonfree parameter and dissipative finite difference scheme proposed by Zhang Hanxin $[34,35]$. This scheme can effectively suppress the oscillation of numerical solutions. The NND format corrects the equation by appropriately adding a third-order term and 
adopts different calculation formats for the upstream and downstream regions of the shock wave:

$$
\left(\frac{\partial \phi}{\partial t}\right)_{j}^{n}+\frac{f_{j+1 / 2 L}^{+n}+f_{j+1 / 2 R}^{-n}}{\partial x}+\frac{f_{j-1 / 2 L}^{+n}+f_{j-1 / 2 R}^{-n}}{\partial x}=S .
$$

As $h_{j+(1 / 2)}^{n}=f_{j+(1 / 2) L}^{+n}+f_{j+(1 / 2) R}^{-n}$ and $h_{j-(1 / 2)}^{n}=f_{j-(1 / 2) L^{+}}^{+n}$ $f_{j-(1 / 2) R}^{-n}$, the changed difference format can be expressed as follows:

$$
\begin{aligned}
\left(\frac{\partial \phi}{\partial t}\right)_{j}^{n} & =-\frac{1}{\Delta x}\left(h_{j+\frac{1}{2}}^{n}-h_{j-\frac{1}{2}}^{n}\right)+S \\
f_{j+(1 / 2) L}^{+n} & =f_{j}^{+n}+\frac{1}{2} \min \bmod \left(\Delta f_{j-(1 / 2)}^{+n}, \Delta f_{j-(1 / 2)}^{+n}\right), \\
\Delta f_{j-(1 / 2) L}^{+n} & =f_{j-1}^{+n}+\frac{1}{2} \min \bmod \left(\Delta f_{j-(3 / 2)}^{+n}, \Delta f_{j-(1 / 2)}^{+n}\right), \\
\Delta f_{j+(1 / 2) R}^{-n} & =f_{j+1}^{-n}-\frac{1}{2} \min \bmod \left(\Delta f_{j+(1 / 2)}^{-n}, \Delta f_{j+(3 / 2)}^{-n}\right), \\
f_{j-(1 / 2) R}^{-n} & =f_{j}^{-n}-\frac{1}{2} \min \bmod \left(\Delta f_{j-(1 / 2)}^{-n}, \Delta f_{j+(1 / 2)}^{-n}\right) .
\end{aligned}
$$

The definition of min $\bmod (x, y)$ has the following meaning: when $x$ and $y$ are the same number, $\min \bmod (x, y)$ takes the value of $x$, the element with the smallest absolute value in $y$, and when $x$ and $y$ are different, $\min \bmod (x, y)$ takes a value of zero.

4.2. Numerical Resolution for Nonconservative Equation. As the two-fluid model is a typical nonconservative equation, the NND format needs to be corrected for it. For the improving NND format, the Godunov scheme [18, 36-38] (Saurel and Abgrall 1999a; Saurel and Abgrall 1999b; Saurel and Lemetayer 2001; and Saurel et al. 2003) can be used as follows:

$$
\begin{aligned}
& \frac{\partial \alpha_{1}}{\partial x}=\frac{\left[\left(\alpha_{1}\right)_{j+(1 / 2)}^{*}-\left(\alpha_{1}\right)_{j-(1 / 2)}^{*}\right]}{(2 \cdot \Delta x)}, \\
& \quad\left(\alpha_{1}\right)_{j+(1 / 2)}^{*}=\left(\alpha_{1}\right)_{j+1}^{*}-\left(\alpha_{1}\right)_{j}^{*},\left(\alpha_{1}\right)_{j-(1 / 2)}^{*}=\left(\alpha_{1}\right)_{j}^{*}-\left(\alpha_{1}\right)_{j-1}^{*} .
\end{aligned}
$$

4.3. Boundary Condition. Boundary conditions were constructed by using the method of characteristics. Taking $(\partial \phi / \partial t)+B(\partial \phi / \partial x)=S$ into the form $(\partial \phi / \partial t)+T[\lambda] T^{-1}(\partial \phi / \partial x)=S$ and then using the feature matrix left multiplied the equation, the following equation could be obtained:

$$
\left\{\begin{array}{l}
T^{-1} \frac{\mathrm{D} \phi}{\mathrm{D} t}=T^{-1} S \\
\frac{\partial x}{\partial t}=\lambda .
\end{array}\right.
$$

The equation of the boundary condition could be changed to the following form:

$$
T^{-1}\left(\phi_{P}-\phi_{L}\right) \Delta t=T^{-1} S_{L} \Delta t .
$$

The compatibility equation used the following form for interpolation calculation:

$$
\begin{gathered}
\phi_{R}=\phi_{C}+\frac{\Delta t}{\Delta x(c-u)\left(\phi_{C}-\phi_{B}\right)}, \\
\phi_{L}=\phi_{C}-\frac{\Delta t}{\Delta x(c+u)\left(\phi_{C}-\phi_{A}\right)}, \\
\phi_{L}=\phi_{C}-\frac{\Delta t}{\Delta x(u)\left(\phi_{C}-\phi_{A}\right)} .
\end{gathered}
$$

\section{Unsteady Friction Model}

Accurate prediction of the water hammer cavitation jet in a pipeline system is the basis of prediction in pipeline obstruction and leakage. In a one-dimensional pipeline system, the unsteady friction model and steady friction model are used to predict the reduction of pressure waves.

A one-dimensional pipeline flow resistance model is usually expressed as follows:

$$
J=J_{s}+J_{u},
$$

where $J_{s}$ is the steady friction model. The steady friction can be expressed by the Darcy-Weisbach relation:

$$
J_{u}=\frac{f V|V|}{2 g D}
$$

The value of steady-state flow resistance coefficient is related to the Reynolds number. For pipeline flow, when the Reynolds number $\operatorname{Re}<2320, f=64 / \operatorname{Re}$, and when $\operatorname{Re}>2320$, according to the Colebrook-White formula, the following expression can be obtained:

$$
\frac{1}{\sqrt{f}}=-2 \lg \left(\frac{2.51}{\operatorname{Re} \sqrt{f}}+\frac{K / D}{3.71}\right) .
$$

In general, the steady friction model cannot well simulate the wave attenuation process in transient flow. Therefore, some scholars put forward the unsteady friction model. Among these unsteady friction models, the twocoefficient decay model has the best application as follows:

$$
J_{u}=K u t \frac{\partial u}{\partial t}+K u x \operatorname{Sign}(u) c\left|\frac{\partial u}{\partial x}\right| .
$$

For the values of $K u t$ and $K u x$, one can refer to the literature [31]. The expression of $\partial u / \partial t$ and $\partial u / \partial x$ in the formula is related to the transfer direction of the characteristic line (63) and (64).

Along the positive characteristic,

$$
\begin{gathered}
\frac{\partial u}{\partial x}=\frac{u_{i}^{n}-u_{i-1}^{n}}{\Delta x}, \\
\frac{\partial u}{\partial t}=\frac{u_{i}^{n}-u_{i}^{n-1}}{\Delta t} .
\end{gathered}
$$


Along the negative characteristic,

$$
\frac{\partial u}{\partial x}=\frac{u_{i}^{n}-u_{i+1}^{n}}{\Delta x} \quad \frac{\partial u}{\partial t}=\frac{u_{i}^{n}-u_{i}^{n-1}}{\Delta t} .
$$

As there are few successful cases in the numerical calculation of wave attenuation with cavitation jet. In this paper, the unsteady friction model is partially integrated with the calculation method of the cavitation jet. When the wave experiences 3-5 phases, the friction model gets switch on to the unsteady friction model to guarantee accurate capture of the pressure wave decay.

\section{Experimental Comparison}

6.1. Experimental System 1. The length of the experimental system is $37.2 \mathrm{~m}$. The pipeline has a slope of $2 \mathrm{~m}$. The pipe inner diameter is $22 \mathrm{~mm}$. The pipe wall thickness is $1.6 \mathrm{~mm}$. Poisson's ratio 0.34, Young's modulus is $E=120 \pm 5 \mathrm{GPa}$, and the wave velocity in the pipe is $1321 \mathrm{~m} / \mathrm{s}$. The experiment temperature was 20 . The saturated steam pressure was $2340 \mathrm{~Pa}$. The material of the pipe was copper [39].

There are three experimental schemes:

Experimental scheme 1: the flow rate was $0.28 \mathrm{~m} / \mathrm{s}$, the flow direction was $\mathrm{p} 1-\mathrm{p} 5$, the valve closed time was $0.009 \mathrm{~s}$, the absolute head upstream was $28.332 \mathrm{~m}$, and the test time was $0.7 \mathrm{~s}$.

Experimental scheme 2: the flow rate was $0.40 \mathrm{~m} / \mathrm{s}$, the flow direction was $\mathrm{p} 5-\mathrm{p} 1$, the valve closed time was $0.0095 \mathrm{~s}$, the absolute head upstream was $23.22 \mathrm{~m}$, and the test time was $1.0 \mathrm{~s}$.

Experimental scheme 3: the flow rate was $1.50 \mathrm{~m} / \mathrm{s}$, the flow direction was p5-p1, the valve closed time was $0.009 \mathrm{~s}$, the absolute head upstream was $23.22 \mathrm{~m}$, and the test time was $1.2 \mathrm{~s}$. The experimental program is shown in Table 1, and the schematic diagram of experimental system 1 is shown in Figure 1:

$$
c=\sqrt{\frac{K / \rho}{1+(K D / E e) c^{\prime}}}=\sqrt{\frac{2.19 \times 10^{9} / 998}{1+\left(2.19 \times 10^{9} / 120 \times 10^{9}\right)(0.0221 / 0.0016) \times 1.02}}=1321 \mathrm{~m} / \mathrm{s} .
$$

6.2. Experimental System 2. The length of the experimental system is $36 \mathrm{~m}$. The pipeline has a slope of $1 \mathrm{~m}$. The pipe inner diameter is $19.5 \mathrm{~mm}$. The pipe wall thickness is $1.588 \mathrm{~mm}$. Poisson's ratio 0.34, Young's modulus is $E=119 \pm 5 \mathrm{GPa}$, and the wave velocity in the pipe is $1280 \mathrm{~m} / \mathrm{s}$. The experiment temperature was 23.9. The saturated steam pressure was $2320 \mathrm{~Pa}$. The material of the pipe was copper [7]. The experimental program is shown in Table 2 , and the schematic diagram of experimental system 1 is shown in Figure 2:

$$
c=\sqrt{\frac{K / \rho}{1+(K D / E e) c^{\prime}}}=\sqrt{\frac{2.19 \times 10^{9} / 997.3}{1+\left(2.19 \times 10^{9} / 119 \times 10^{9}\right)(0.01905 / 0.0016) \times 0.88}}=1280 \mathrm{~m} / \mathrm{s} .
$$

\section{Discussion}

The results of the 7- and 5-equation models are compared using these nine experiments. The time-effectiveness, calculation accuracy, residual void of gas, and internal velocity characteristics of the two models are analyzed.

7.1. Time-Effectiveness. The 7-equation model, 5-equation model, and method of characteristic (MOC) $[4,6,7]$ are selected for the comparison for time-effectiveness. The time step and the number of grids are guaranteed equality in the calculation. Nine experiments were chosen for comparative verification. The calculated time is statistically calculated and plotted as shown in Figure 3. In Figure 3, MOC is used as the measurement standard of unit 1 , and the data in the 7 equation model and the 5-equation model are multiples of the calculation time of the MOC.

7.2. Calculation Accuracy. According to the calculation principle of the MOC method, it does not have the capacity to carry out accurate numerical simulation of the two-phase flow when cavitation jet occurs. Therefore, the calculation accuracy of the MOC is low. However, the MOC is easily programmable, so it has high time-effectiveness.

The calculation results of the three models are compared, as shown in Figures 4-7. In the simulation of 1-3 wave phases, both 7-equation and 5-equation models could well capture the wave changes of jet cavitation.

It can be seen that the 5-equation model has a good calculation time-effectiveness on the basis of guaranteeing the calculation accuracy.

7.3. Residual Void of Gas. The main reason for the inaccurate calculation of the model is the residual void of the gas. So the distribution void of fraction in the 7-equation model and 5equation model is presented. As shown in Figures 8 and 9, the 5-equation model can better eliminate residual void of the gas after each iteration. From equations (13), (14) and (40), (41), it can be seen that the 5-equation model reduce the interference between phase parameters and 
TABLe 1: Experimental program.

\begin{tabular}{lccc}
\hline Experimental scheme & Velocity $(\mathrm{m} / \mathrm{s})$ & Initial water head $(\mathrm{m})$ & Theoretical head rise $(\mathrm{m})$ \\
\hline 1 & 0.280 & 28.33 & 37.69 \\
2 & 0.400 & 23.22 & 53.84 \\
3 & 1.500 & 22.66 & 201.90 \\
\hline
\end{tabular}

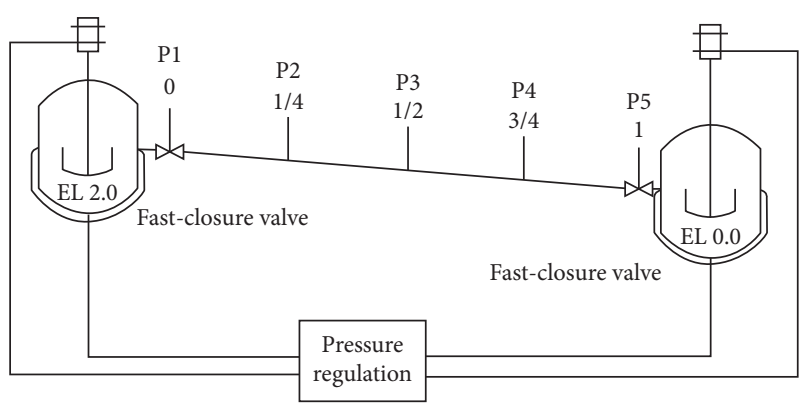

FIgURE 1: Schematic diagram of experimental system 1.

TABLE 2: Experimental program.

\begin{tabular}{lcccc}
\hline $\begin{array}{l}\text { Experimental } \\
\text { scheme }\end{array}$ & $\begin{array}{c}\text { Velocity } \\
(\mathrm{m} / \mathrm{s})\end{array}$ & $\begin{array}{c}\text { Initial } \\
\text { water } \\
\text { head }(\mathrm{m})\end{array}$ & $\begin{array}{c}\text { Valve } \\
\text { closed } \\
\text { time }(\mathrm{s})\end{array}$ & $\begin{array}{c}\text { Theoretical } \\
\text { head rise }(\mathrm{m})\end{array}$ \\
\hline 4 & 0.332 & 33.55 & 0.03608 & 43.33 \\
5 & 0.401 & 33.54 & 0.01848 & 52.34 \\
6 & 0.466 & 33.32 & 0.03520 & 60.82 \\
7 & 0.696 & 33.05 & 0.02640 & 90.84 \\
8 & 0.938 & 36.68 & 0.03608 & 122.43 \\
9 & 1.125 & 31.88 & 0.04400 & 146.83 \\
\hline
\end{tabular}

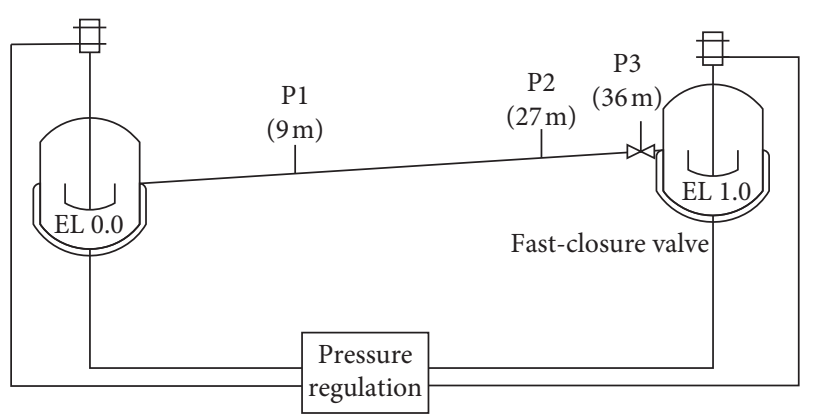

FIgURE 2: Schematic diagram of experimental system 2.

thermodynamic parameters, making the void of fraction simulation more accurate.

7.4. Lag Time Characteristic Analysis. The internal response curves of pressure, velocity, and void of fraction of schemes 3 and 9 were taken for analysis. So the wave phase and period of normal launching cavitation jet are taken for theoretical analysis. In Table 3, the wave velocity is considered as a constant value.

It can be seen in Table 3 that the larger the intensity of the cavitation jet is, the longer the lag time (deviation) of the wave is. The variation trend of velocity and pressure near the

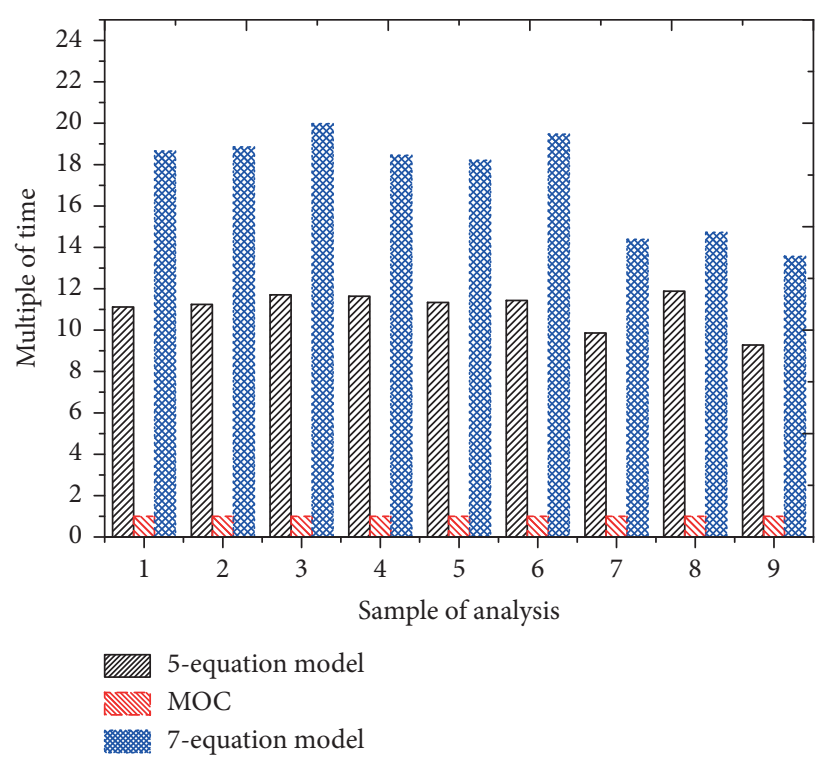

FIGURE 3: Comparison diagram of computing time-effectiveness of the three models.

tail position is displayed (under the 5-equation model), as shown in Figures 10-13.

As shown in Figures 12 and 13, the internal velocity distribution forming is divided into six stages. The six calculation schemes are, respectively, divided into the following five processes for the calculation scheme of cavitation-free jet:

(1) In the reverses stage of 1-2, the liquid starts to move in the opposite direction of the flow, and the pressure starts to gradually decrease

(2) In the recoveries stage of 2-3, the backflow starts to slow down gradually, and the velocity starts to gradually reduce and stops after reaching 0

(3) In the stabilizations stage of 3-4, the velocity is 0

(4) In the acceleration stage of 4-5, it begins to accelerate in the same direction with the initial flow, and the pressure starts to gradually recover

(5) In the deceleration stage of 5-6, the velocity gradually decreases, and pressure gradually increases

The above five stages are repeated until the water hammer wave attenuation disappears.

As shown in Figures 10 and 11, the internal velocity distribution of the formation of the cavitation jet is divided into seven stages, and the six processes are as follows:

(1) In the stage of 1-2 flow reverses, the liquid starts to move in the opposite direction of the flow direction, and the pressure starts to gradually decrease. 


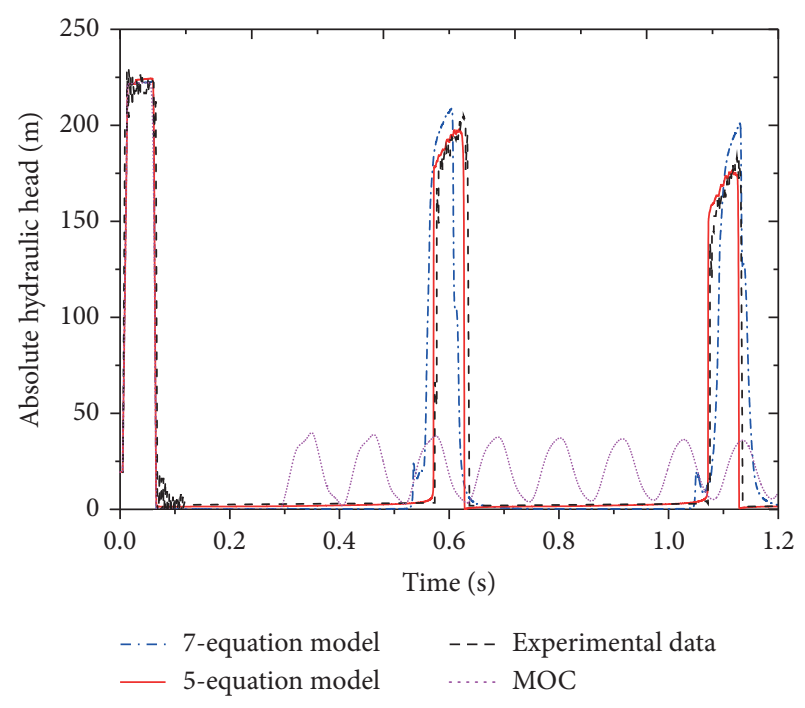

Figure 4: Comparison of scheme 3 calculation scheme (endpoint of the system).

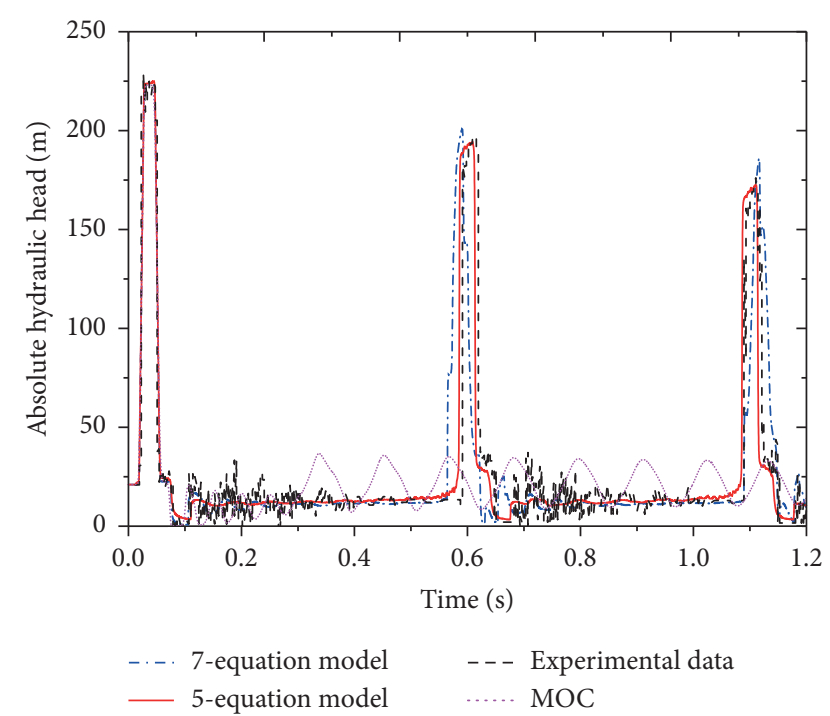

FIGURE 5: Comparison of scheme 3 calculation scheme (midpoint of the system).

(2) In the stage of 2-3 flow recoveries, the backflow began to slow down gradually, and the velocity gradually decreased but does not return to 0 . The void of the cavitation jet is still forming.

(3) In the stage of 3-4 flow recoveries and acceleration, the velocity in the first half slows down, while in the second half accelerates. In the whole process, the slope is greater than in stage 2-3. At this point, the void generated by the cavitation jet starts to disappear rapidly, and the pressure is assured to remain unchanged.

(4) In the gentle flow stage of 4-5, the slope of the forward accelerated flow is lower and the duration of time is shorter than that in the 3-4 stage.

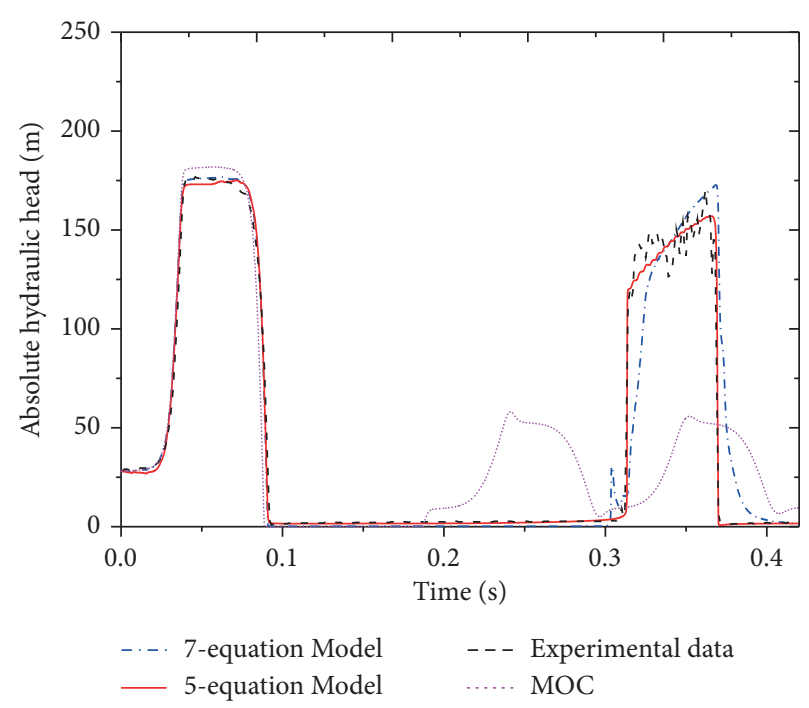

FIgURE 6: Comparison of scheme 9 calculation scheme (endpoint of the system).

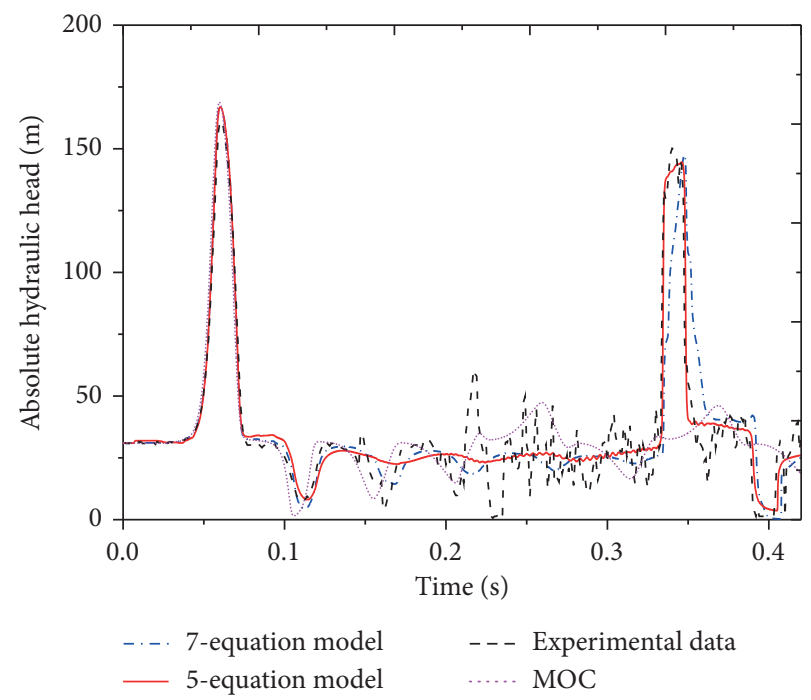

FIGURE 7: Comparison of scheme 9 calculation scheme (a quarter of the system).

(5) In the acceleration stage of 5-6, it continues to accelerate in a positive direction, and the pressure is guaranteed to remain unchanged.

(6) In the flow deceleration stage of 6-7, the velocity gradually decreases and pressure gradually increases.

It can be seen that the main differences lie in the 3-4 stage and $4-5$ stage, in which the velocity increases significantly, leading to the generation of voids and the lag of time characteristics in the calculation.

7.5. Analysis of Pressure Amplitude Characteristics. It can be seen from the difference in the distribution of 3-4, 4-5, and 5-6 stages on the curve that these three stages play relatively different roles in the change of cavitation. The two-fluid 


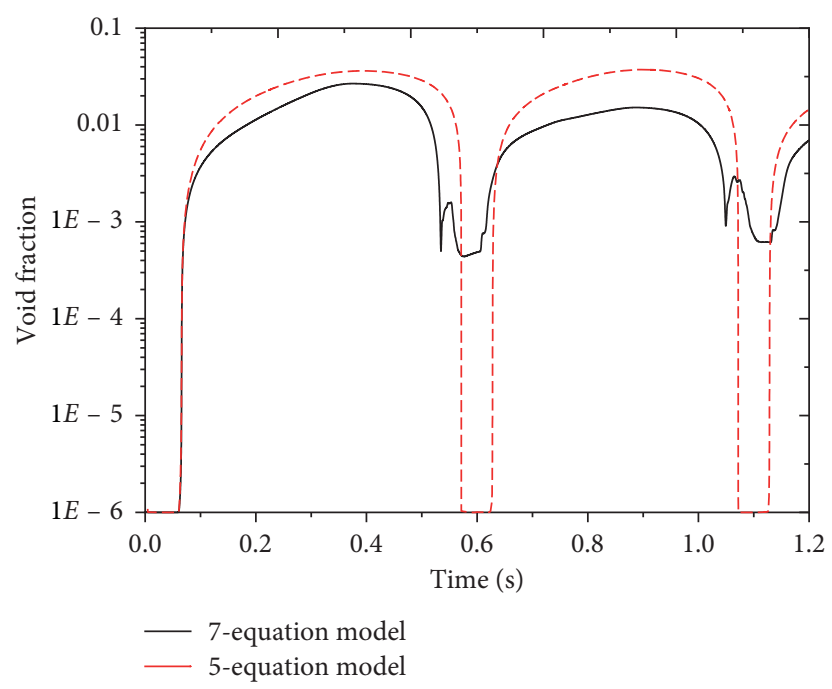

FIgURE 8: Comparison of the cavitation rate of scheme 3 (endpoint of the system).

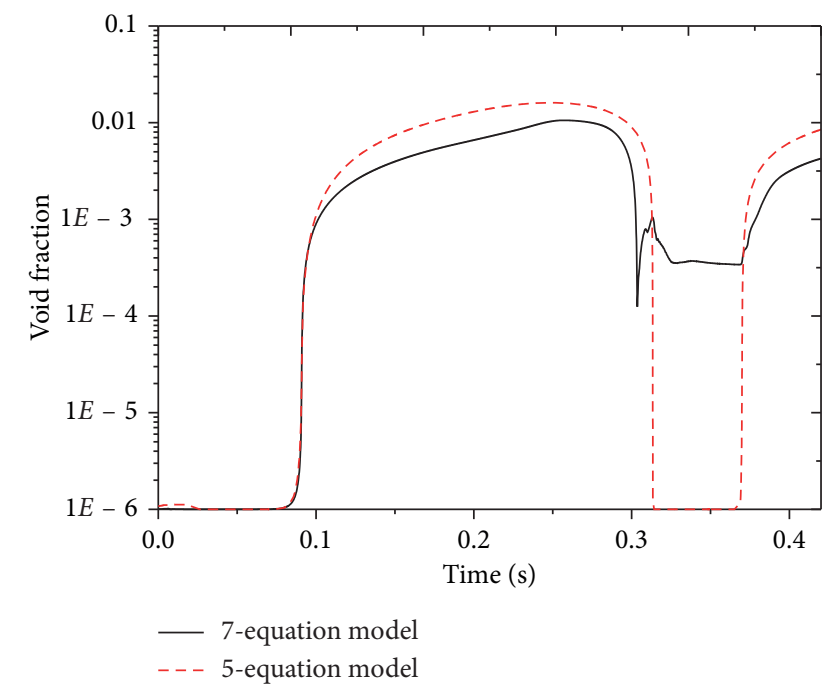

Figure 9: Comparison of the cavitation rate of scheme 9 (endpoint of the system).

TABLE 3: Wave phase and cycle of scheme 3 and scheme 9

\begin{tabular}{lccccc}
\hline Scheme & $\begin{array}{c}\text { Wave } \\
\text { velocity } C \\
(\mathrm{~m} / \mathrm{s})\end{array}$ & $\begin{array}{c}\text { Wave } \\
\text { phase } \\
2 \mathrm{~L} / \mathrm{c}(\mathrm{s})\end{array}$ & $\begin{array}{c}\text { Wave } \\
\text { cycle } \\
4 \mathrm{~L} / \mathrm{c}(\mathrm{s})\end{array}$ & $\begin{array}{c}\text { The } \\
\text { actual } \\
\text { cycle }(\mathrm{s})\end{array}$ & $\begin{array}{c}\text { Deviation } \\
(\mathrm{s})\end{array}$ \\
\hline 3 & 1320 & 0.0564 & 0.1128 & 0.5713 & 0.4585 \\
9 & 1280 & 0.0563 & 0.1125 & 0.3133 & 0.2008 \\
\hline
\end{tabular}

model is taken as an example, where the transport equation of the void can be written as follows:

$$
\frac{\partial \alpha_{1}}{\partial t}+u_{I} \nabla \cdot \alpha_{1}=\mu\left(P_{1}-P_{2}\right)+\frac{\dot{m}}{\rho_{I}} .
$$

When only considering the mathematical properties of two-phase coupling (ignoring variables such as viscosity and surface tension), the generation of the void is generated from the following two aspects:

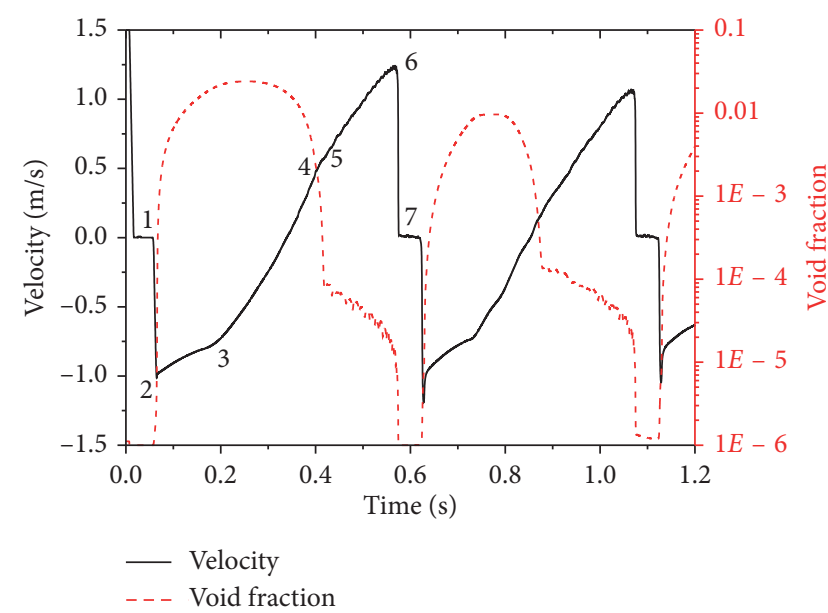

FIgURE 10: Change of internal velocity in scheme 3.

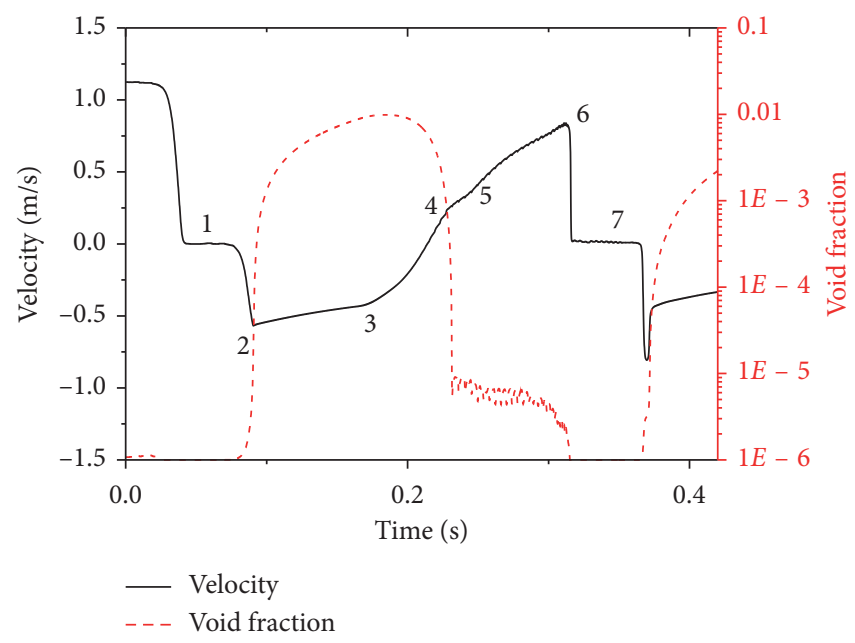

FIgURE 11: Change of internal velocity in scheme 9. 


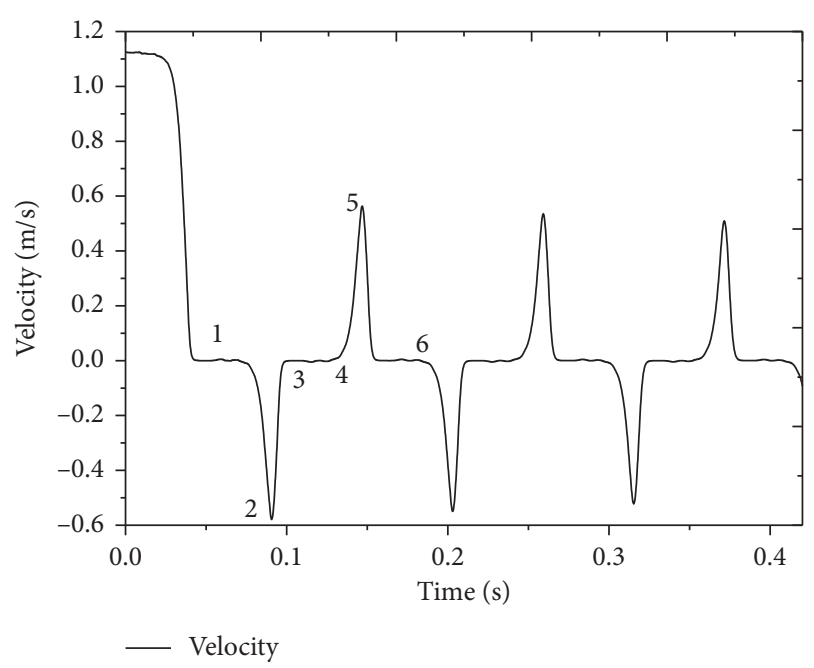

FIGURE 12: The internal velocity of the calculation scheme with no cavitation jet.

$\mu\left(P_{1}-P_{2}\right)$ : gas volume changes due to two-phase pressure changes

$\dot{m} / \rho_{I}$ : the change of cavity volume caused by phase transition

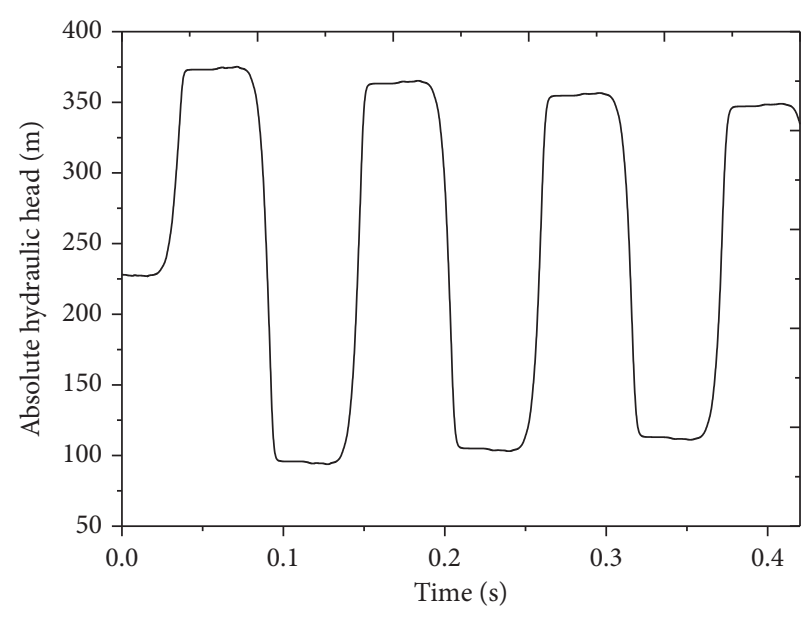

FIGURE 13: The internal pressure of the calculation scheme with no cavitation jet.

It is considered that at the end of each calculation, the two-phase pressure tends to be equal, which is $P_{1}^{*}-P_{2}^{*}=0$, the relaxation coefficient of the pressure changes as follows:

$$
\begin{gathered}
\mu=\frac{1}{\left[\left(\left(P_{I}-\rho_{2}^{2} \partial e_{2} / \partial \rho_{2}\right) /\left(\alpha_{2} \rho_{2} \partial e_{2} / \partial P_{2}\right)\right)-\left(\left(P_{I}-\rho_{1}^{2} \partial e_{1} / \partial \rho_{1}\right) /\left(\alpha_{1} \rho_{1} \partial e_{1} / \partial P_{1}\right)\right)\right] \Delta t}, \\
\mu\left(P_{1}-P_{2}\right)=\frac{\left(P_{1}-P_{2}\right)}{\left[\left(\left(P_{I}-\rho_{2}^{2} \partial e_{2} / \partial \rho_{2}\right) /\left(\alpha_{2} \rho_{2} \partial e_{2} / \partial P_{2}\right)\right)-\left(P_{I}-\rho_{1}^{2} \partial e_{1} / \partial \rho_{1}\right) /\left(\alpha_{1} \rho_{1} \partial e_{1} / \partial P_{1}\right)\right] \Delta t} .
\end{gathered}
$$

The above equations are expressed as the change of gas void caused by the change of pressure.
The change of cavity volume caused by phase transition is expressed as follows:

$$
\dot{m} / \rho_{I}=\frac{2 S / R}{\left[\left(\left(P_{I}-\rho_{2}^{2} \partial e_{2} / \partial \rho_{2}\right) /\left(\alpha_{2} \rho_{2} \partial e_{2} / \partial P_{2}\right)\right)-\left(\left(P_{I}-\rho_{1}^{2} \partial e_{1} / \partial \rho_{1}\right) /\left(\alpha_{1} \rho_{1} \partial e_{1} / \partial P_{1}\right)\right) /\left(\alpha_{1} \rho_{1} \partial e_{1} / \partial P_{1}\right)\right] \Delta t}
$$

where $2 S / R$ is usually greater than $\left(P_{1}-P_{2}\right)$. According to the slope of the curve, it can be concluded that the 4-5 stage is usually the stage of cavitation condensation and collapse, while the 5-6 stage is the gas volume change caused by the two-phase pressure.

It can be found that in the experimental scheme 1 with low cavitation intensity (theoretical head change $43.33 \mathrm{~m}$ ), the actual pressure head change reaches $67.58 \mathrm{~m}$, but in the experimental scheme 6 with low cavitation intensity (theoretical head change $146.83 \mathrm{~m}$ ), the actual pressure head change is only $144.98 \mathrm{~m}$. Therefore, the internal flow characteristics of the two curves were analyzed separately, as shown in Figures 14 and 15.

It can be seen in Figure 14 that the 4-5 stage of change in the internal characteristics of velocity is steeper. Stage 4-5 corresponds to the elimination stage of cavitation. The main reason for high pressure is that the cavitation formed in a small size, so it quickly collapses under high pressure. After cavitation collapse, the high-pressure liquid forms jet flow, which rapidly impacts the wall and causes the pressure to increase instantly.

However, it is different from the velocity distribution trend in Figure 14 (scheme 1). The change rate of stage 45 more flattens than that in stages 3-4 and 5-6 in Figure 15 . In scheme 6 , the cavitation volume is larger, which results in longer elimination time. The slope is smaller, and the pressure increase is not as large as in scheme 1 . This explains the reasons why the intensity of the cavitation jet is low and the pressure rise is high. The pressure difference between phases in Figures 16 and 17 also confirms the phenomenon that after cavitation collapse, high-pressure liquid forms jet flow and rapidly 


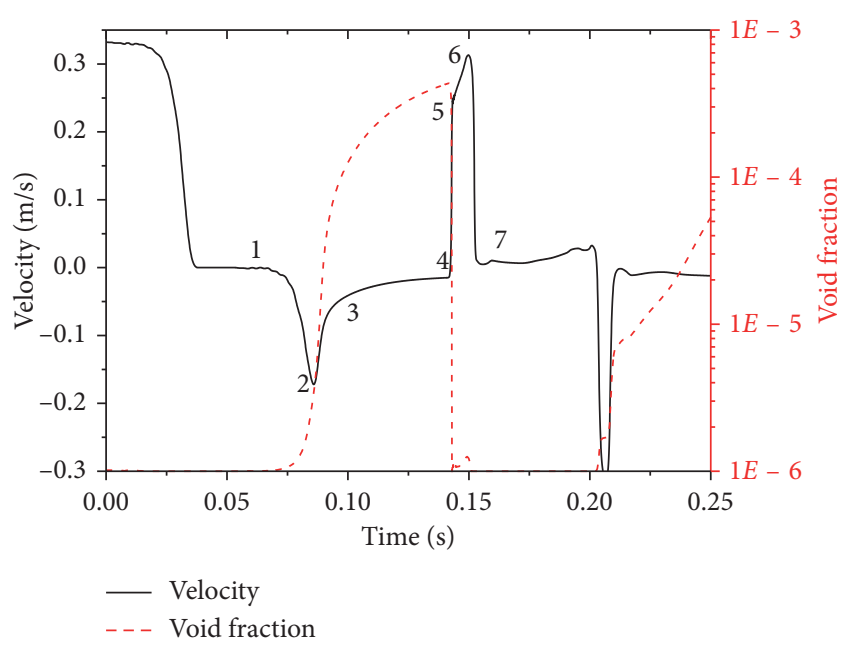

FIGURE 14: Scheme 1 calculation of internal velocity change of the scheme.

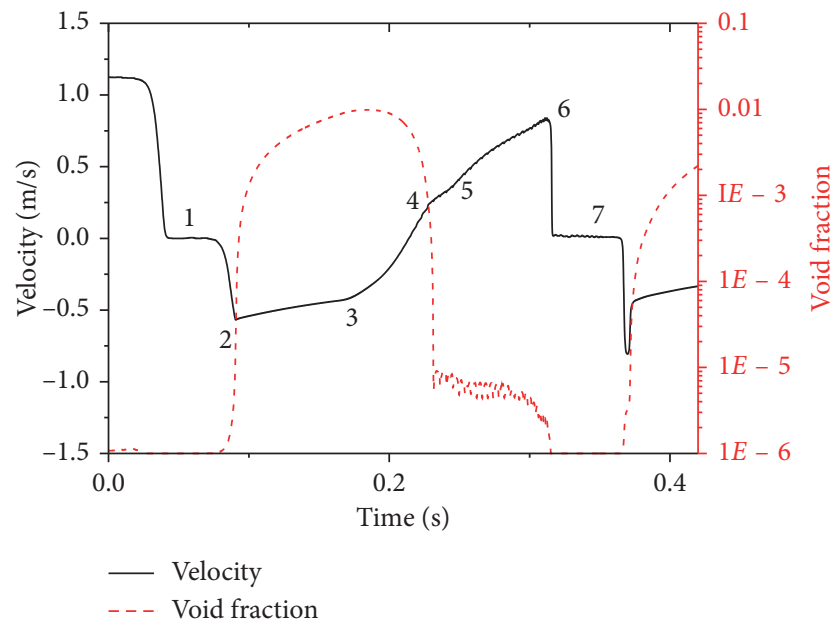

FIGURE 15: Scheme 6 calculation of internal velocity change of the scheme.

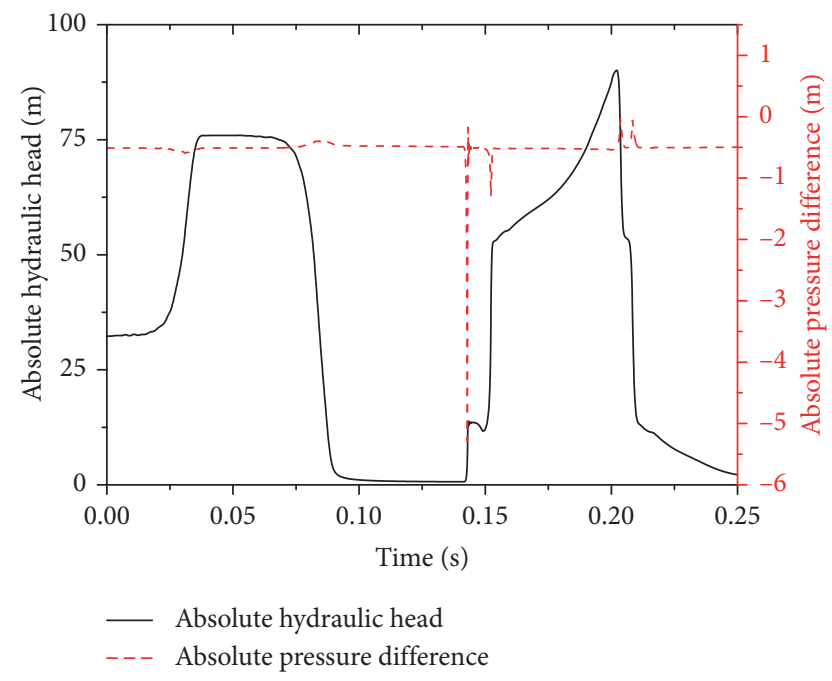

Figure 16: Comparison of scheme 1 pressure calculation scheme and pressure difference between two phases.

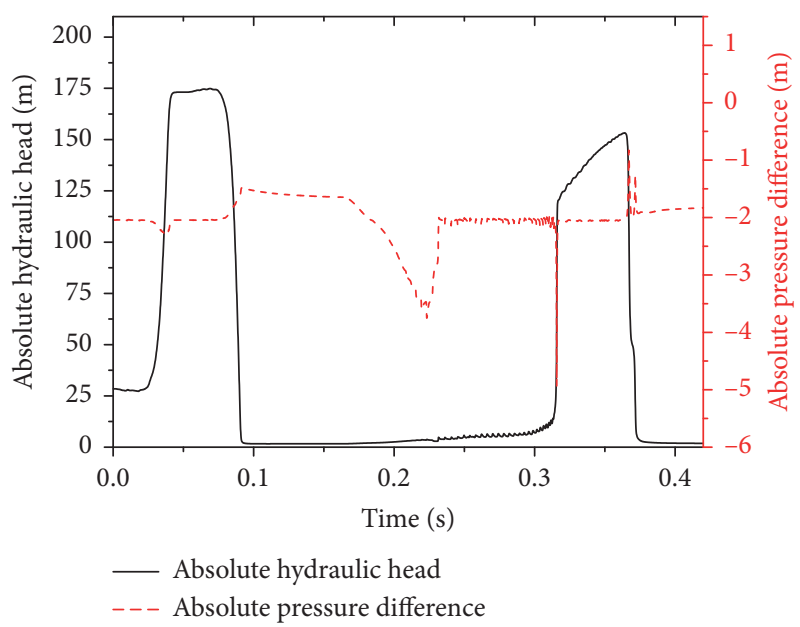

Figure 17: Comparison of scheme 6 pressure calculation scheme and pressure difference between two phases.

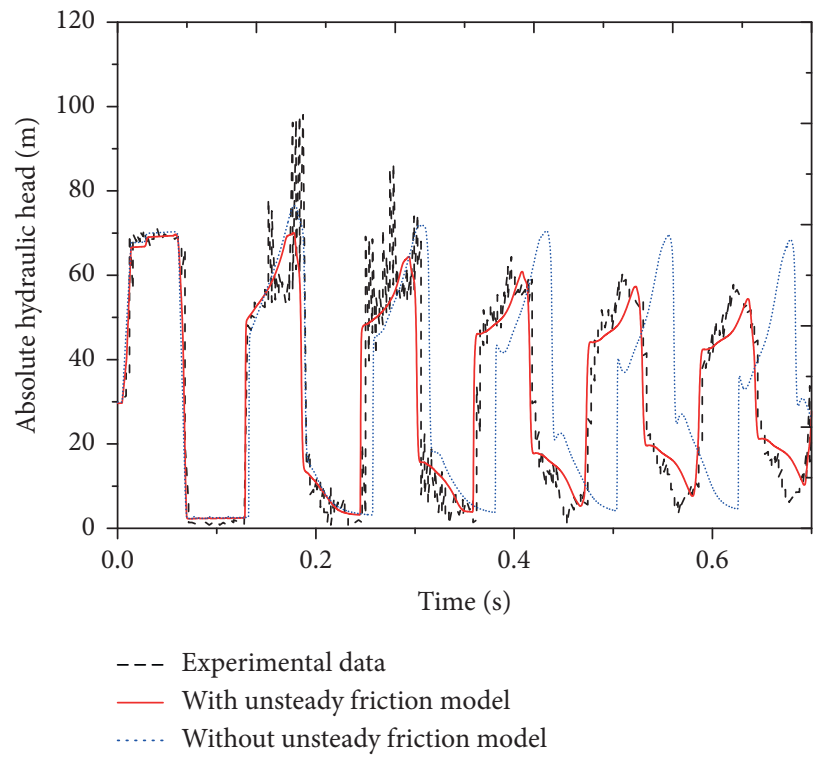

FIGURE 18: Comparison of calculation results of scheme 7 with and without unsteady flow friction model (endpoint of the system).

impacts the wall, leading to an instantaneous increase in pressure.

Stage 5-6 corresponds to the gas compression stage. Since the gas modulus is larger than that of the water vapor, the slope of the 5-6 phase is lower.

7.6. Unsteady Friction Model. When the steady flow resistance model is adopted, only the first 2-3 waves of cavitation jet pressure can be predicted accurately. The whole attenuation and propagation process of the pressure wave cannot be analyzed and demonstrated in detail. Therefore, it is necessary to point out the unsteady flow resistance model to the calculation method for analysis. Calculation examples of scheme 7 and scheme 8 are adopted for verification.

In calculation scheme 7 , the following are observed: 


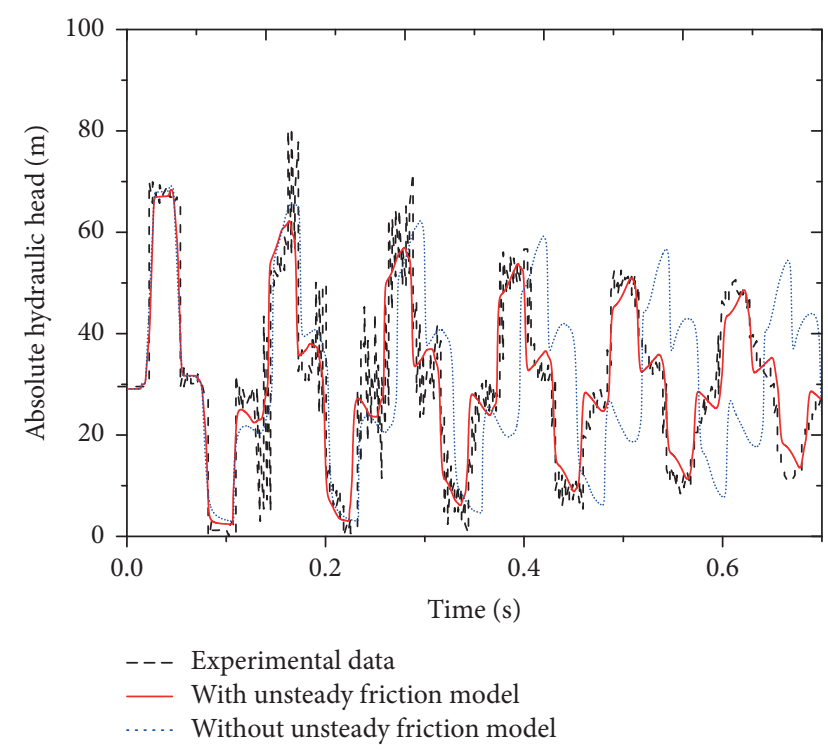

FIgURE 19: Comparison of calculation results of scheme 7 with and without unsteady flow friction model (midpoint).

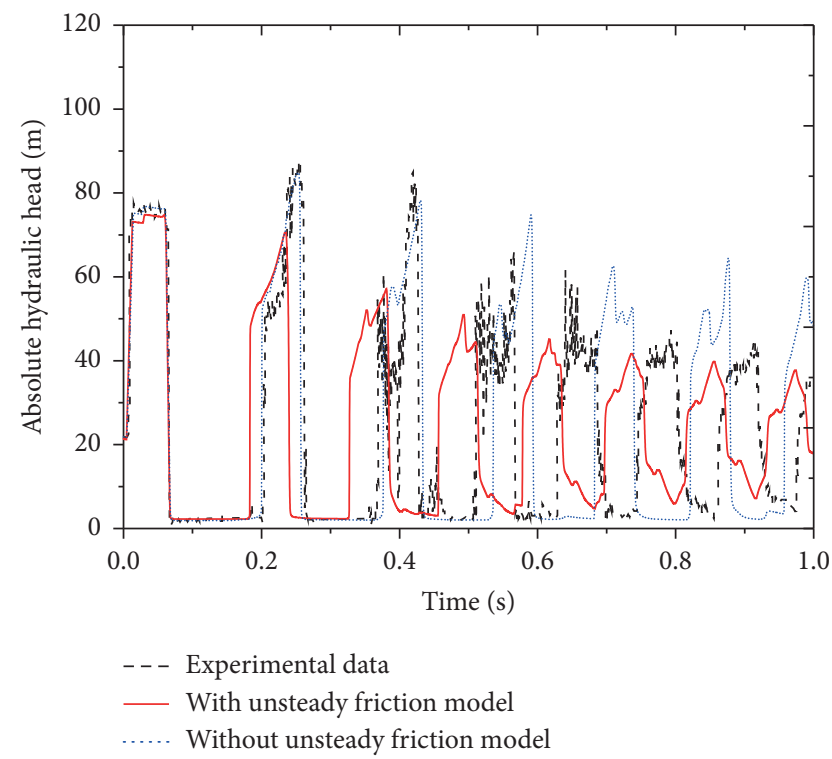

FIgUre 20: Comparison of calculation results of scheme 8 with and without unsteady flow friction model (endpoint of the system).

(1) In the calculation method without the addition of an unsteady flow friction model, the simulation accuracy of first and second pressure waves is good, but the subsequent pressure attenuation process is far from the experiment

(2) In the calculation method with the addition of an unsteady flow friction model, the amplitude deviation of the first and second pressure wave simulation is relatively large, but the subsequent pressure attenuation process is in good agreement with the experiment

In calculation scheme 8 , the following are observed:

(1) In the calculation method without the addition of an unsteady flow friction model, the simulation

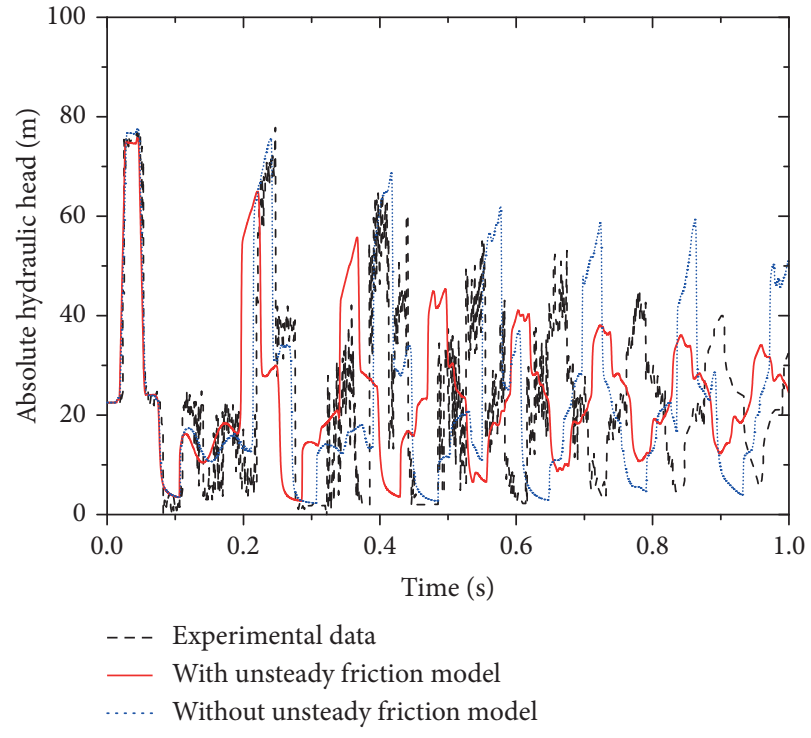

FIGURE 21: Comparison of calculation results of scheme 8 with and without unsteady flow friction model (midpoint).

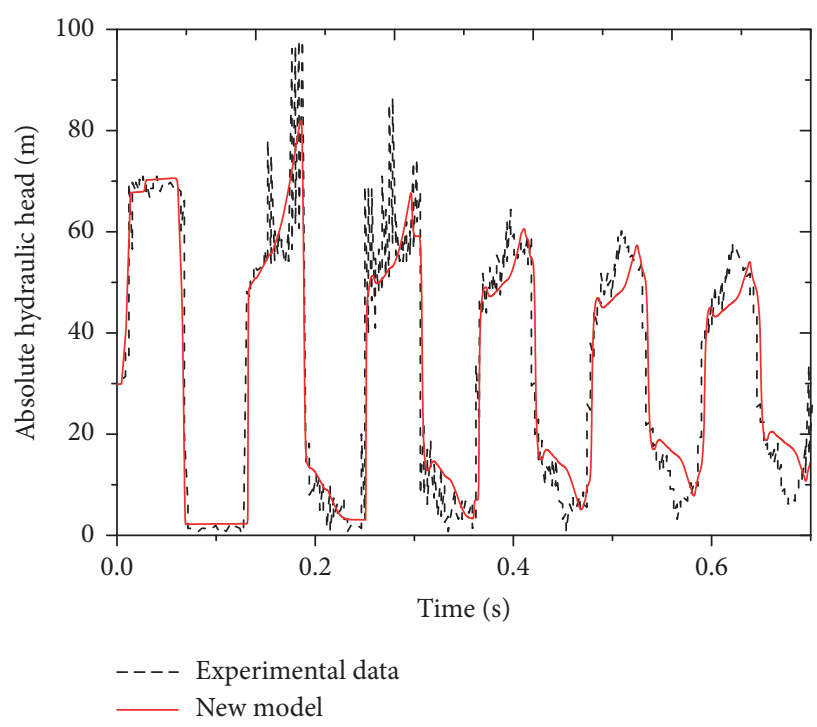

FIGURE 22: Comparison of calculation results of scheme 7 improved unsteady flow friction model (endpoint of the system).

accuracy of the first and second pressure waves is good, but the subsequent pressure attenuation process is more different from the experiment

(2) In the calculation method with an unsteady flow friction model, the simulated amplitude and phase of the first and secondary pressure waves have deviated, but the subsequent pressure attenuation process is higher than the experimental value

In conclusion, for the process of pipeline cavitation jet, the applicability of the two-coefficient unsteady flow resistance model decreases as the severity of cavitation jet increases. Therefore, the phase deviation and the calculation error of the pressure attenuation process are caused. To 


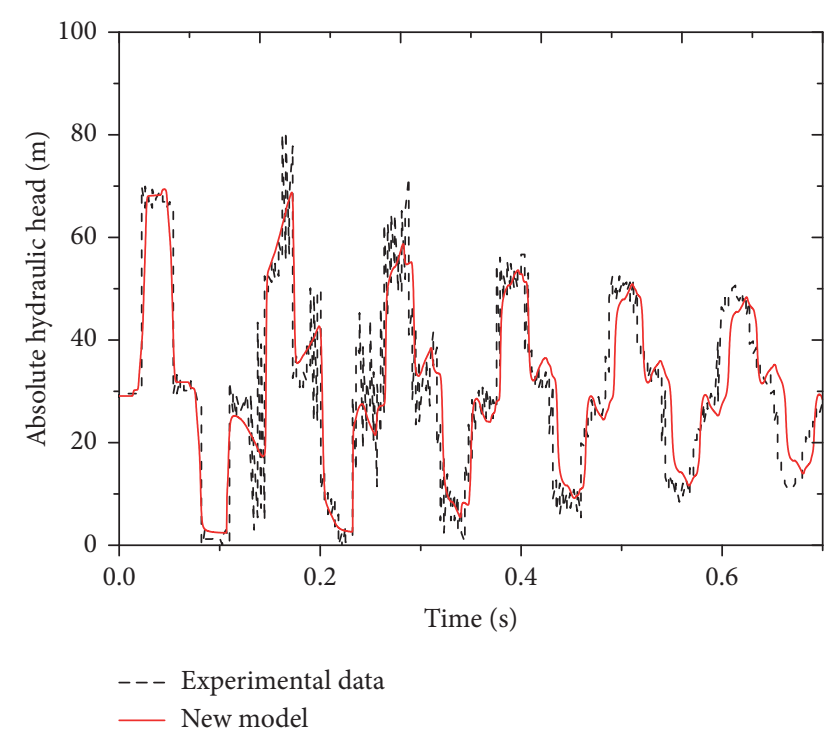

Figure 23: Comparison of calculation results of scheme 7 improved unsteady flow friction model (midpoint).

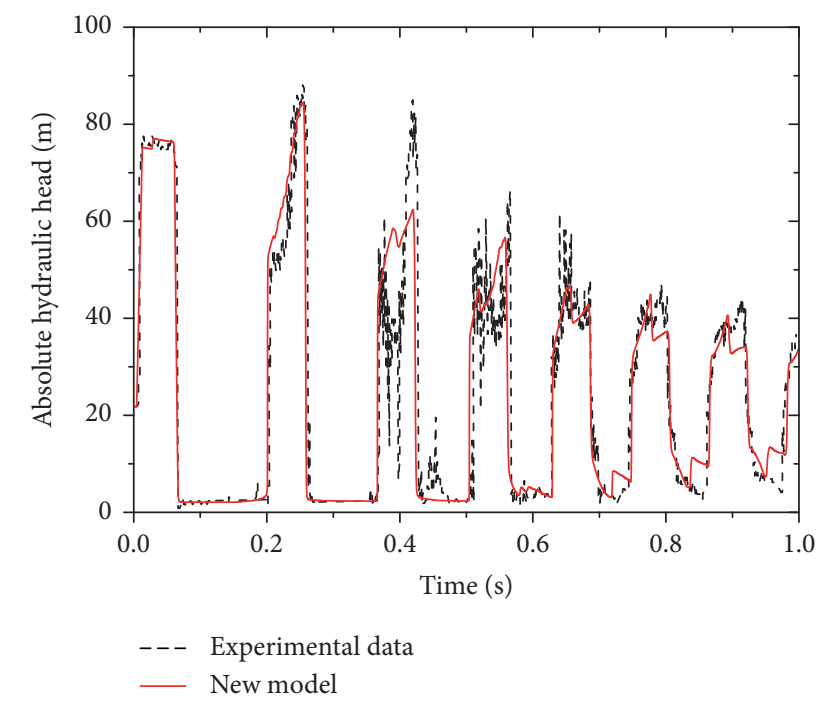

Figure 24: Comparison of calculation results of scheme 8 improved unsteady flow friction model (endpoint of the system).

simulate the full attenuation process of cavitation jet pressure wave reasonably, the double-coefficient flow resistance model needs to be re-integrated.

7.7. Application of Improved Transient Resistance Model. It can be seen from Figures 18 and 19 and Figures 20 and 21 that to achieve accurate simulation of the process with cavitation jet, a switch needs to be set in the calculation. This switch does not turn on the unsteady flow resistance model after $3-4^{*}(2 \mathrm{~L} / \mathrm{c})$ (wave phase). In this case, the model can not only meet the pressure and phase accuracy requirements of the first-two peaks but also ensure a good simulation of pressure wave attenuation. The simulation results are shown in Figures 22-25.

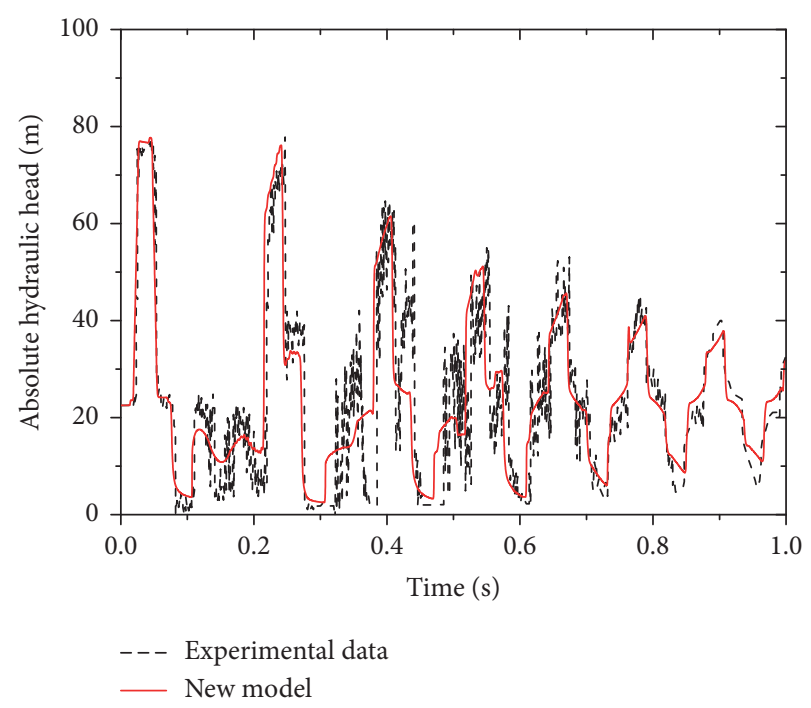

Figure 25: Comparison of calculation results of scheme 8 improved unsteady flow friction model (midpoint).

\section{Conclusion}

(1) In this paper, a more efficient simplified numerical calculation method is established by simplifying the seven-equation two-fluid model, which not only has high computational efficiency but also can further eliminate the problems existing in the two-fluid model.

(2) It is found that when a cavitation jet is formed, the velocity flow characteristics are different from the traditional ones. These differences result in different response time and response amplitude of water hammer pressure.

(3) Coupled with the two-coefficient transient flow friction model, a numerical calculation method suitable for predicting the attenuation of the cavitation jet pressure wave is found. By opening the transient friction model when the water hammer wave transmits 3-5 phases, this method can simulate the reduction of wave well. It provides a reference for the research of pressure pipeline leakage, vibration, and noise.

\section{Data Availability}

All experimental data and calculated data that support the findings of this study are available from the corresponding author upon reasonable request.

\section{Conflicts of Interest}

The authors declare that they have no conflicts of interest.

\section{Acknowledgments}

The authors gratefully acknowledge the financial support provided by Gansu Province Natural Science Foundation of China (No. 1208RJYA023). 


\section{References}

[1] J. P. T. Kalkwijk and C. Kranenburg, "Cavitation in horizontal pipelines due to water hammer," Journal of the Hydraulics Division, vol. 97, pp. 1585-1605, 1971.

[2] G. A. Provoost, "Investigation into cavitation in a prototype pipeline caused by water hammer," in Proceedings of the 2 nd International Conference on Pressure Surges, vol. 78, London, UK, September 1976.

[3] M. A. Chaiko, "A finite-volume approach for simulation of liquid-column separation in pipelines," Journal of Fluids Engineering, vol. 128, no. 6, pp. 1324-1335, 2006.

[4] C. Kranenburg, "Transient cavitation in pipelines. Civil Engineering \& Geosciences," Doctoral thesis, Delft University of Technology, Deparentment of civil engineering, Laboratory of Fluid Mechanics, Delft, Netherlands, 1974.

[5] P. H. Schweitzer and V. G. Szebehely, "Gas evolution in liquids and cavitation," Journal of Applied Physics, vol. 21, no. 12, pp. 1218-1224, 1950.

[6] A. Bergant, A. R. Simpson, and A. S. Tijsseling, "Water hammer with column separation: a historical review," Journal of Fluids and Structures, vol. 22, no. 2, pp. 135-171, 2006.

[7] A. R. Simpson, Large water hammer pressures due to column separation in sloping pipes, Ph.D. thesis, The University of Michigan, Ann Arbor, MI, USA, 1986.

[8] A. Adamkowski and M. Lewandowski, "Investigation of hydraulic transients in a pipeline with column separation," Journal of Hydraulic Engineering, vol. 138, no. 11, pp. 935-944, 2012.

[9] J. Jiang, Y. Fu, L. Zhang, Y. Li, W. Ji, and Y. Liu, "The investigation of gas-liquid two-phase transient flow based on steger-warming flux vector splitting method in pipelines," Advances in Mechanical Engineering, vol. 8, no. 10, 2016.

[10] Y. Fu, J. Jiang, Y. Li, and R. Ying, "Calculation method of relaxation coefficient in two-fluid water hammer calculation model," Huazhong Keji Daxue Xuebao (Ziran Kexue Ban)/ Journal of Huazhong University of Science and Technology (Natural Science Edition), vol. 46, no. 10, pp. 51-56, 2018.

[11] F. Crouzet, F. Daude, P. Galon, J.-M. Hérard, O. Hurisse, and Y. Liu, "Validation of a two-fluid model on unsteady liquidvapor water flows," Computers \& Fluids, vol. 119, pp. 131-142, 2015.

[12] D. Furfaro and R. Saurel, "Modeling droplet phase change in the presence of a multi-component gas mixture," Applied Mathematics and Computation, vol. 272, pp. 518-541, 2016.

[13] Y. Fu, J. Jiang, Y. Li, and R. Ying, "Water hammer simulated method based on two-fluid model in two-phase flow," Huazhong Keji Daxue Xuebao (Ziran Kexue Ban)/Journal of Huazhong University of Science and Technology (Natural Science Edition), vol. 46, no. 1, pp. 126-132, 2018.

[14] F. You, J. Jin, L. Yanhui, and Y. Rui, "Calculation of pipe water hammer pressure with liquid column separation by improved two-fluid model," Transactions of the Chinese Society of Agricultural Engineering, vol. 34, no. 15, pp. 58-65, 2018.

[15] R. Saurel, F. Petitpas, and R. Abgrall, "Modelling phase transition in metastable liquids: application to cavitating and flashing flows," Journal of Fluid Mechanics, vol. 607, pp. 313-350, 2008.

[16] H. Lochon, F. Daude, P. Galon, and J.-M. Hérard, "Comparison of two-fluid models on steam-water transients," ESAIM: Mathematical Modelling and Numerical Analysis, vol. 50, no. 6, pp. 1631-1657, 2016.

[17] M. Pelanti and K.-M. Shyue, "A Roe-type scheme with low Mach number preconditioning for a two-phase compressible flow model with pressure relaxation," Bulletin of the Brazilian Mathematical Society, New Series, vol. 47, no. 2, pp. 655-669, 2016.

[18] R. Saurel, A. Chinnayya, and F. Renaud, "Thermodynamic analysis and numerical resolution of a turbulent-fully ionized plasma flow model," Shock Waves, vol. 13, no. 4, pp. 283-297, 2003.

[19] R. Saurel, E. Franquet, E. Daniel, and O. Le Metayer, "A relaxation-projection method for compressible flows. Part I: the numerical equation of state for the Euler equations," Journal of Computational Physics, vol. 223, no. 2, pp. 822-845, 2007.

[20] F. Petitpas, E. Franquet, R. Saurel, and O. Le Metayer, "A relaxation-projection method for compressible flows. Part II: artificial heat exchanges for multiphase shocks," Journal of Computational Physics, vol. 225, no. 2, pp. 2214-2248, 2007.

[21] R. Saurel, F. Petitpas, and R. A. Berry, "Simple and efficient relaxation methods for interfaces separating compressible fluids, cavitating flows and shocks in multiphase mixtures," Journal of Computational Physics, vol. 228, no. 5, pp. 16781712, 2009.

[22] R. Saurel and F. Petitpas, "A hyperbolic non equilibrium model for cavitating flows," Fluid Dynamics of Cavitation and Cavitating Turbopumps, pp. 279-316, Springer, Berlin, Germany, 2007.

[23] E. B. Shuy, "Wall shear stress in accelerating and decelerating turbulent pipe flows," Journal of Hydraulic Research, vol. 34, no. 2, pp. 173-183, 1996.

[24] A. E. Vardy and J. M. B. Brown, "Transient, turbulent, smooth pipe friction," Journal of Hydraulic Research, vol. 33, no. 4, pp. 435-456, 1995.

[25] G. Pezzinga, "Quasi-2D model for unsteady flow in pipe networks," Journal of Hydraulic Engineering, vol. 125, no. 7, pp. 676-685, 1999.

[26] W. Zielke, "Closure to "discussions of "frequency dependent friction in transient pipe flow"” (1968, ASME J. Basic eng., 90, pp. 413-414)," Journal of Basic Engineering, vol. 90, no. 3, p. $414,1968$.

[27] A. E. Vardy and K.-L. Hwang, "A weighting function model of transient turbulent pipe friction," Journal of Hydraulic Research, vol. 31, no. 4, pp. 533-548, 1993.

[28] B. Brunone, B. W. Karney, M. Mecarelli, and M. Ferrante, "Velocity profiles and unsteady pipe friction in transient flow," Journal of Water Resources Planning and Management, vol. 126, no. 4, pp. 236-244, 2000.

[29] A. E. Vardy and J. M. B. Brown, "Transient turbulent friction in smooth pipe flows," Journal of Sound and Vibration, vol. 259, no. 5, pp. 1011-1036, 2003.

[30] B. Kucienska, J.-M. Seynhaeve, and M. Giot, "Friction relaxation model for fast transient flows application to water hammer in two-phase flow-the WAHA code," International Journal of Multiphase Flow, vol. 34, no. 2, pp. 188-205, 2008.

[31] H. Prashanth Reddy, W. F. Silva-Araya, and M. Hanif Chaudhry, "Estimation of decay coefficients for unsteady friction for instantaneous, acceleration-based models," Journal of Hydraulic Engineering, vol. 138, no. 3, pp. 260-271, 2011.

[32] M.-H. Lallemand, A. Chinnayya, and O. Le Metayer, "Pressure relaxation procedures for multiphase compressible flows," International Journal for Numerical Methods in Fluids, vol. 49, no. 1, pp. 1-56, 2005.

[33] A. Zein, M. Hantke, and G. Warnecke, "Modeling phase transition for compressible two-phase flows applied to 
metastable liquids," Journal of Computational Physics, vol. 229, no. 8, pp. 2964-2998, 2010.

[34] H. Zhang and F. Zhuang, "Nnd schemes and their applications to numerical simulation of two- and three-dimensional flows," Advances in Applied Mechanics, vol. 29, no. 193, 1992.

[35] X. Zhang, L. Qiu, H. Qi, X. Zhang, and Z. Gan, "Modeling liquid hydrogen cavitating flow with the full cavitation model," International Journal of Hydrogen Energy, vol. 33, no. 23, pp. 7197-7206, 2008.

[36] R. Saurel and R. Abgrall, "A simple method for compressible multifluid flows," SIAM Journal on Scientific Computing, vol. 21, no. 3, pp. 1115-1145, 1999.

[37] R. Saurel and R. Abgrall, "A multiphase Godunov method for compressible multifluid and multiphase flows," Journal of Computational Physics, vol. 150, no. 2, pp. 425-467, 1999.

[38] R. Saurel and O. Lemetayer, "A multiphase model for compressible flows with interfaces, shocks, detonation waves and cavitation," Journal of Fluid Mechanics, vol. 431, pp. 239-271, 2001.

[39] A. Bergant and A. R. Simpson, "Water hammer and column separation measurements in an experimental apparatus," University of Adelaide, Department of Civil and Environmental Engineering, Adelaide, Australia, 1995. 